\title{
Proof-of-Concept Testing of a Sustained Vortex-Flow Configuration for Hybrid Rocket Motors
}

\author{
Ray Wilkinson ${ }^{1}$, Ken Hart ${ }^{2}$, and Rodney Day ${ }^{3}$ \\ University of Hertfordshire, Hatfield, AL10 9AB, UK \\ and \\ Ian Coxhill ${ }^{4}$ \\ AMPAC-ISP (UK) Limited, Aylesbury, HP18 0NZ, UK
}

\begin{abstract}
One of the drawbacks of hybrid rocket motors is the limited regression rate of the fuel grain, which impacts on the scalablility of the type. A number of methods of increasing this have been proposed and successfully tested, in particular the use of swirling oxidiser flow to create turbulence and increase the local oxidiser mass flux. However, many of these have limitations in practical motors for many applications, and a common problem is that of the swirl decaying within the motor. The proposed method described here is to use a tapering fuel-grain port to sustain the swirl. A short series of firings indicates that such a method may be effective, although the evidence is limited thus far. The majority of previous research has used gaseous oxygen, and it has been found during this test programme that using liquid nitrous oxide poses particular problems. However, solutions have been found, and are described in this paper.
\end{abstract}

\section{Introduction}

$\mathrm{H}$ ybrid motors can offer a number of significant advantages over other forms of propulsion for small launch vehicles and for satellite propulsion. This is particularly applicable to the fledgling space-tourism industry, where the benefits of low cost and high safety can be usefully exploited. However, hybrid motors suffer from a number of drawbacks that have yet to be overcome, mainly as a result of the low level of development they have enjoyed in comparison with composite (solid) and liquid engines. The volumetric efficiency is low, leading to increased vehicle size and structural mass, and much of the ongoing research is aimed at improving the fuel regression rate in an attempt to improve volumetric efficiency. A further limitation is that motor configurations do not scale predictably, meaning that sub-scale laboratory tests are of limited applicability, and tests at full scale are needed to determine empirical corrections to optimise the design. The relatively fixed regression rate also determines the maximum practical size for hybrid motors, currently limited to about 250,000lb thrust. It therefore appears unlikely that hybrid motors will be used for large launch vehicles in the foreseeable future, and indicates complex and inefficient fuel-grain geometries for motors near the upper end of practical sizes. However, the use of a nitrous oxide/HTPB hybrid motor in SpaceShipOne and SpaceShipTwo gives impetus to the development of this type.

A promising area of research is to encourage better mixing of boundary-layer flow in the combustion chamber, hence improved heat transfer to the fuel surface, by generating a vortex. This is commonly induced by introducing a tangential component to the oxidiser injection, or by aerodynamic means such as cutting flutes within the fuel grain to induce helical flow. ${ }^{1}$ Some researchers have experimented with different motor configurations, such as Surrey Satellite Technologies' so-called 'pancake hybrid" ${ }^{2}$ and Knuth et al.'s coaxial vortex design, ${ }^{3,4}$ with some success. Majdalani and Vyas also published at least one paper on this technique. All sources report a considerable increase in regression rate - up to seven times the rate for non-swirling hybrids. However, each has its own problems - with simple swirling injection, expansion of the gases and the liberation of fuel vapour from the surface cause the helix to decay quickly; with fluted fuel grains the flutes erode as the fuel grain regresses; in the SSTL hybrid, the swirl decayed within half

${ }^{1}$ Principal Lecturer, School of Engineering and Technology, Member AIAA.

${ }^{2}$ Principal Lecturer, School of Engineering and Technology.

${ }^{3}$ Principal Lecturer, School of Engineering and Technology.

${ }^{4}$ Chief Engineer, Member AIAA.

American Institute of Aeronautics and Astronautics 


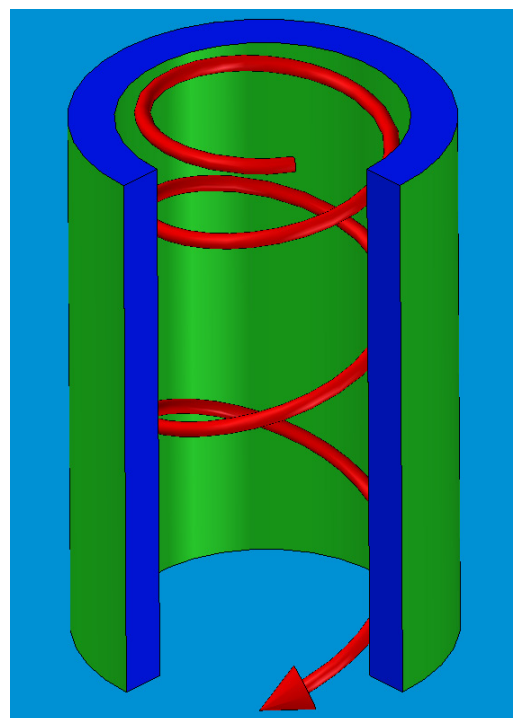

a.

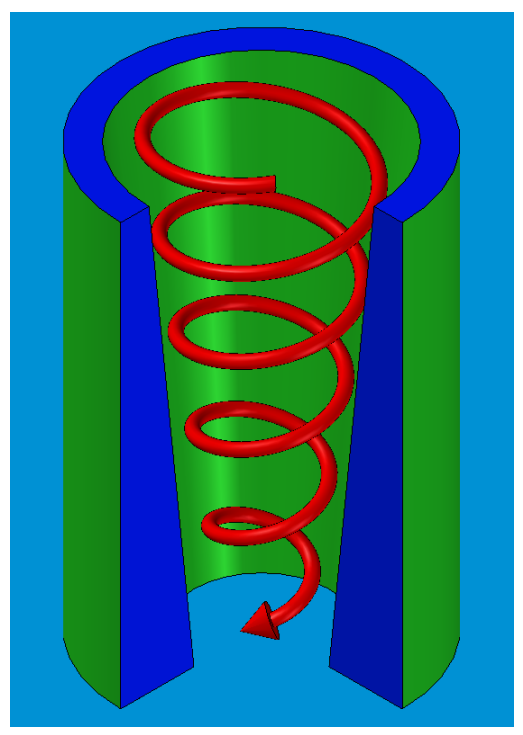

b.

Figure 1. Swirling injection in straight and tapered cavities. In a fuel grain with a standard, parallel-sided cavity (a), swirl decays as the gases pass down the cavity. If the cavity is tapered axially $(b)$, conservation of angular momentum accelerates the tangential velocity component; if the taper is optimised, then the swirl angle will be precisely maintained.

a rotation; and the coaxial design produces low volumetric efficiency and a complex grain design and/or injection system. A method is therefore being sought to sustain helical flow simply and effectively.

Another drawback of many hybrids is that the relatively slow combustion and the need for a reasonable oxidiser mass flux require a long combustion chamber, leading to slender motors and consequent difficulties in vehicle design for many applications. Alternative configurations have been tried by using a pair of flat disc-shaped fuel grains with tangential oxidiser injection (the so-called 'pancake hybrid'), but it was found that the swirl decayed quickly, with the majority of the flow being radial, thus reducing the path length and oxidiser 'stay' time within the motor. (However, the primary aim with this configuration was to use the centrifugal effects to hold the denser, uncombusted material inside the motor for longer, to improve combustion efficiency.) The shape of these pancake motors is also inconvenient for some applications, especially for launch vehicles, due to the large diameter required.

\section{Decay Mechanism}

The decay of the vortex in swirl-injection hybrids is considered here to arise from three principal causes - expansion of the gases during combustion, wall friction and viscous losses, and fuel mass addition. Taking these in turn:

\section{A. Expansion of gases}

The swirl of the gases within the combustion chamber can be separated into an axial velocity component and a tangential velocity component. The relative magnitudes of these two define the amount of swirl, which could be expressed either as the angle of the flow to the motor primary axis, or as a swirl number as used by various researchers, principally based on the work of Kitoh ${ }^{6}$. The gases under consideration consist of a mixture of oxidiser and fuel vapour, both uncombusted, and also the products of combustion that has already taken place. This combustion will add considerable heat into the gases, raising their temperature. The pressure within the combustion chamber must be higher at the head end than at the nozzle, in order for flow towards the nozzle to take place. However, it is assumed here that the axial pressure distribution within the combustion chamber is reasonably constant compared to its absolute value, so it may be considered that this gradient is relatively small. Thus the heat addition to the gases results in a large temperature rise at approximately constant pressure, producing a large volume change. This volume change thus requires that the axial velocity of the gases increases with axial distance. 
Since there can be no net flow radially for a combustion chamber of constant diameter, there will be no tangential velocity change. The result of these two effects is that the swirl will be reduced as the relative magnitudes of the two components, axial and tangential, change. This mechanism is similar in nature to the change from turbulent to substantially laminar flow within a liquid bi-propellant engine as the gases accelerate towards the nozzle.

\section{B. Wall friction and viscous losses}

For (non-combusting) swirling flow in pipes, the decay of swirl has been found to be related to wall-surface roughness and to Reynolds number, with smoother walls and higher Reynolds number causing a lower rate of decay. It might be reasoned that surface blowing, as present in a hybrid motor, would form a barrier between the swirling gases and the wall surface, largely eliminating the surface friction, in the way that an air table works. Despite the obvious surface roughness during the burn, this effect will therefore be assumed to be equivalent to having a very smooth wall, and therefore negligible in comparison to the other effects.

\section{Fuel mass addition}

The gases within the combustion chamber have an angular momentum as a result of the tangential velocity component. However, since only the oxidiser is initially swirled, the angular momentum is fixed by the oxidiser massflow rate and the angle at which the oxidiser is introduced. The fuel vapour liberated as a result of heat transfer to the fuel grain surface will initially travel normal to the surface, and then be entrained in the same direction as the prevailing gas flow through viscous shear. However, no additional angular momentum will be introduced, so the mass addition will reduce the mean tangential velocity component, and hence reduce swirl.

\section{Sustained Vortex-Flow Concept}

Research at the University of Hertfordshire has generated a concept of a fuel-grain with a conical, narrowing cavity, together with the induction of helical flow through tangential oxidiser injection. The principle is that, when optimised, this conical configuration creates an accelerating effect on the flow, through conservation of angular momentum, which will offset the decay caused by the other mechanisms. The initial testing reported here, to prove the concept, compares different flow configurations within a hybrid motor of nominally $150-250 \mathrm{~N}$ thrust. The propellants used were nitrous oxide and polypropylene, a combination that is very appropriate to space-tourism applications. Testing was carried out in four configurations - axial flow through two injector ports into an untapered fuel-grain cavity (control configuration), axial flow through two injector ports into a tapered fuel-grain cavity, helical injection through two inclined injector ports into an untapered fuel-grain cavity, and helical injection through two inclined injector ports into a tapered fuel-grain cavity. This arrangement of tests permits the effects of helical flow and any enhancement due to the taper to be separated. To prove the concept, tests took place in the spring and early summer of 2010.

Once viability of the concept has been fully demonstrated, future research will aim to optimise the cavity configuration and nozzle matching to permit a detailed analysis of the full benefits achievable. This requires morecomprehensive instrumentation to determine propellant flows and pressures, and measurement of combustion-chamber temperatures and flow patterns, and sufficient testing to determine the statistical variation. The ultimate aim is to characterise the concept for a range of motor sizes and propellant combinations. A flight-capable motor in the range $300-1000 \mathrm{~N}$ thrust will also be built and flown, to evaluate any issues associated with residual vortex flow in the exhaust plume.

\section{Test Configurations}

Four combinations of motor configuration were tested, as described above. The planned order of testing was selected to minimise the extent of reconfiguration - removing and replacing the fuel grain requires very little dismantling compared to the replacement of the injector. In addition, a number of repeated configurations were planned to determine the repeatability of identical tests. However the order of testing was changed as the test programme developed.

The original design of swirling injector introduced the oxidiser at an angle of $42^{\circ}$ to the motor axis, which was the greatest angle achievable whilst maintaining adequate thickness of the injector plate. The second design of swirling injector allowed this to be increased to $45^{\circ}$. The taper angle of the fuel grain port was $2.5^{\circ}$, which corresponded to a ratio between maximum and minimum diameters of approximately 2 . The mean port diameter of the tapered-port fuel grain was the same as that of the parallel grain.

Fuel-grain geometry for these comparisons offered a number of options. Ideally, the mass flux for all tests would be identical, since it is widely accepted that regression rate is proportional to some exponent of mass flux, originally 
proposed by Marxman and Gilbert to be 0.8 (Ref. 8). In practice, this exponent varies from one motor to another, and is determined empirically. It was not possible to guarantee the flow rates through injectors of varying designs would be identical, nor that the pressure of the nitrous oxide supply, which is highly sensitive to ambient temperature, would be constant between tests. Further, with swirling flow, the effective grain port area is reduced, and this would be expected to increase regression rate regardless of any other effects. Since the fuel grain port in question is also tapered, the matching between the tapered and untapered grains becomes somewhat arbitrary, and the decision was made to make the mean diameters of the two variants equal. The length of the combustion chamber limits the taper of the grain port to 2.5 degrees if a reasonable wall thickness is to be preserved. If the concept shows promise and the regression rate is increased significantly, it should be possible to shorten the grain and hence increase the taper angle.

\section{Test Setup}

The testing was carried out in the University's custom-built rocket-motor test cell. The test setup consisted of a motor with a combustion chamber of $50 \mathrm{~mm}$ bore and $250 \mathrm{~mm}$ long, with a twin-port injector. This injector has two principal variants - one with two injector ports parallel to the axis of the motor and one with two ports set at angles of 42 degrees (first version) or 45 degrees (later versions) to the motor axis, to induce swirling flow. The motor has a solenoid valve of $3 \mathrm{~mm}$ bore to control oxidiser flow, and has an additional port through which gaseous oxygen, for ignition, and gaseous nitrogen, for purging, can be fed into the combustion chamber. The motor is mounted on thin steel flexures, and bears against a load cell at the head end. Since the flexures impart some stiffness to the mountings, a pair of pulleys are mounted in line with the motor axis, allowing weights to be attached to simulate thrust. This arrangement provides for calibration of the load cell/flexure arrangement. Four lengths of M3 threaded bar tie the motor together, using pairs

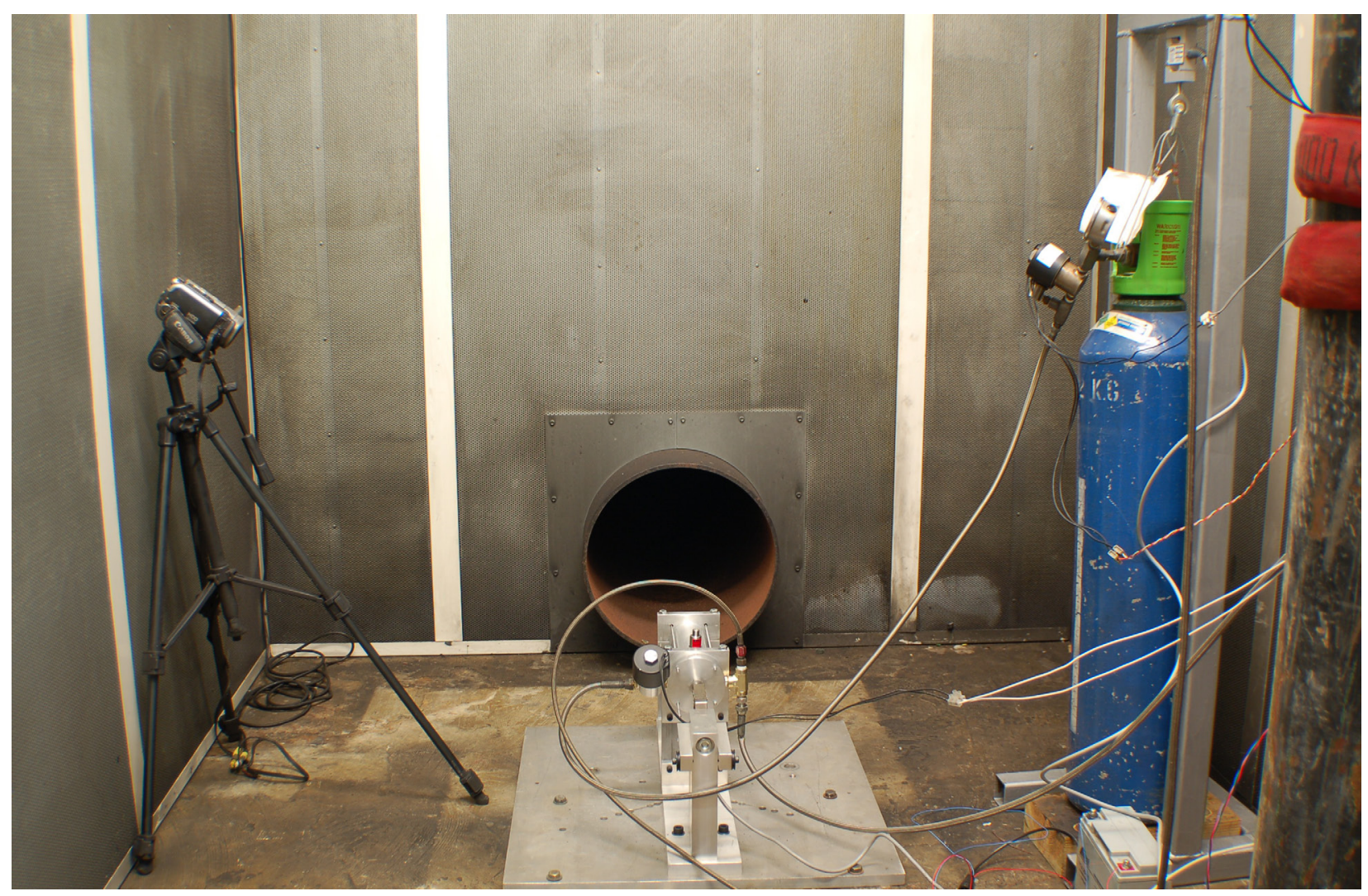

Figure 2. Test-cell setup. The motor is attached to a plate bolted to the test-cell floor. A 20-litre nitrous-oxide cylinder is suspended from a load cell to permit continuous monitoring of the oxidiser flow rate. Note the camera used to monitor the firings, before being fitted with a protective cover. Oxygen (for starting) and nitrogen (purge) cylinders are just out of picture. 
of nuts at each end. These are designed, and tested in a tensile test machine, to fail if the combustion chamber pressure exceeds 1000psi, allowing the aft closure to be ejected in a safe direction. A commercial graphite nozzle is used, and the design oxidiser flow rate is approximately matched to the nozzle throat area. If the combustion products were to contain significant quantities of unreacted oxygen, it is likely that severe nozzle erosion would be experienced, and it may then be necessary to replace the graphite nozzle with a cooled copper nozzle. However, severe erosion would indicate that the internal configuration is not optimal, and may necessitate a redesign. In the event, erosion was generally small, and nozzles needed to be replaced infrequently (two nozzles were used for a total of 16 firings); there was, however, some variation in erosion between firing configurations.

Oxidiser flow rate was measured by suspending the supply cylinder, of 20 litres capacity and weighing approximately $50 \mathrm{~kg}$ full, from a frame fitted with a load cell. Output from

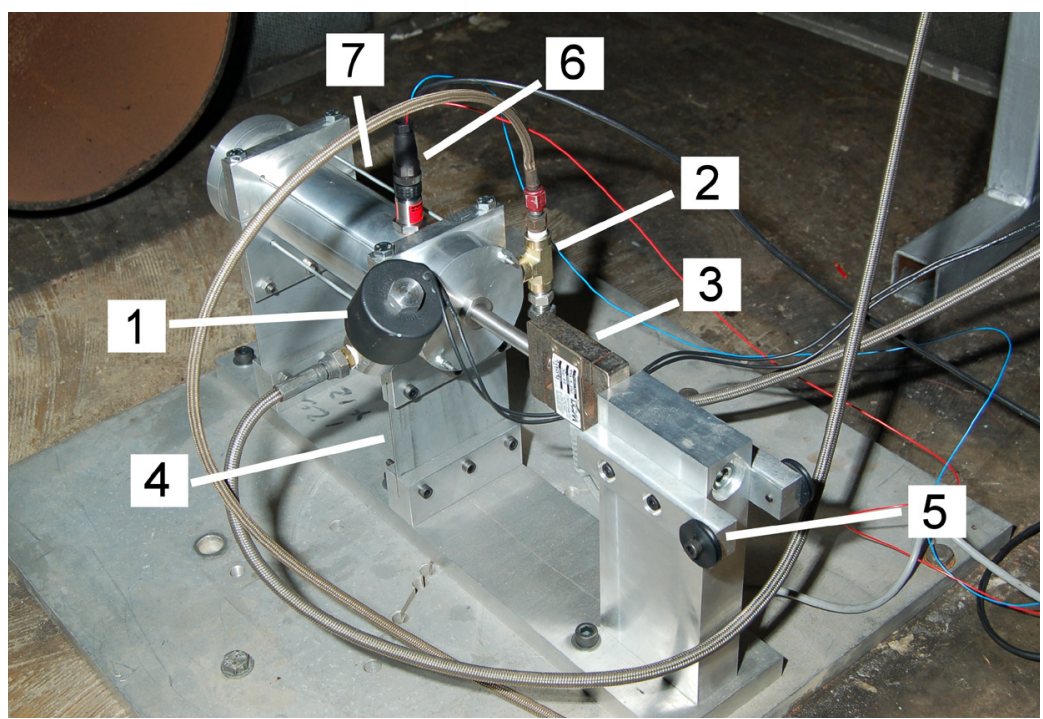

Figure 3. Test motor. Nitrous oxide feed to the motor is controlled by a solenoid valve (1), with an oxygen supply for start-up and a nitrogen feed for purging connected to a second inlet port via a tee (2). Both feeds are controlled by solenoid valves at the cylinders. Thrust is measured using a load cell (3), and the motor is mounted on steel flexures (4). Calibration is carried out using weights, attached in line with the load cell using a Kevlar lanyard running over pulleys (5). Chamber pressure is measured using a pressure sensor (6) close to the injector. Four ties (7), of M3 threaded bar, are set to fail and release the aft closure if safe pressure limits are exceeded.

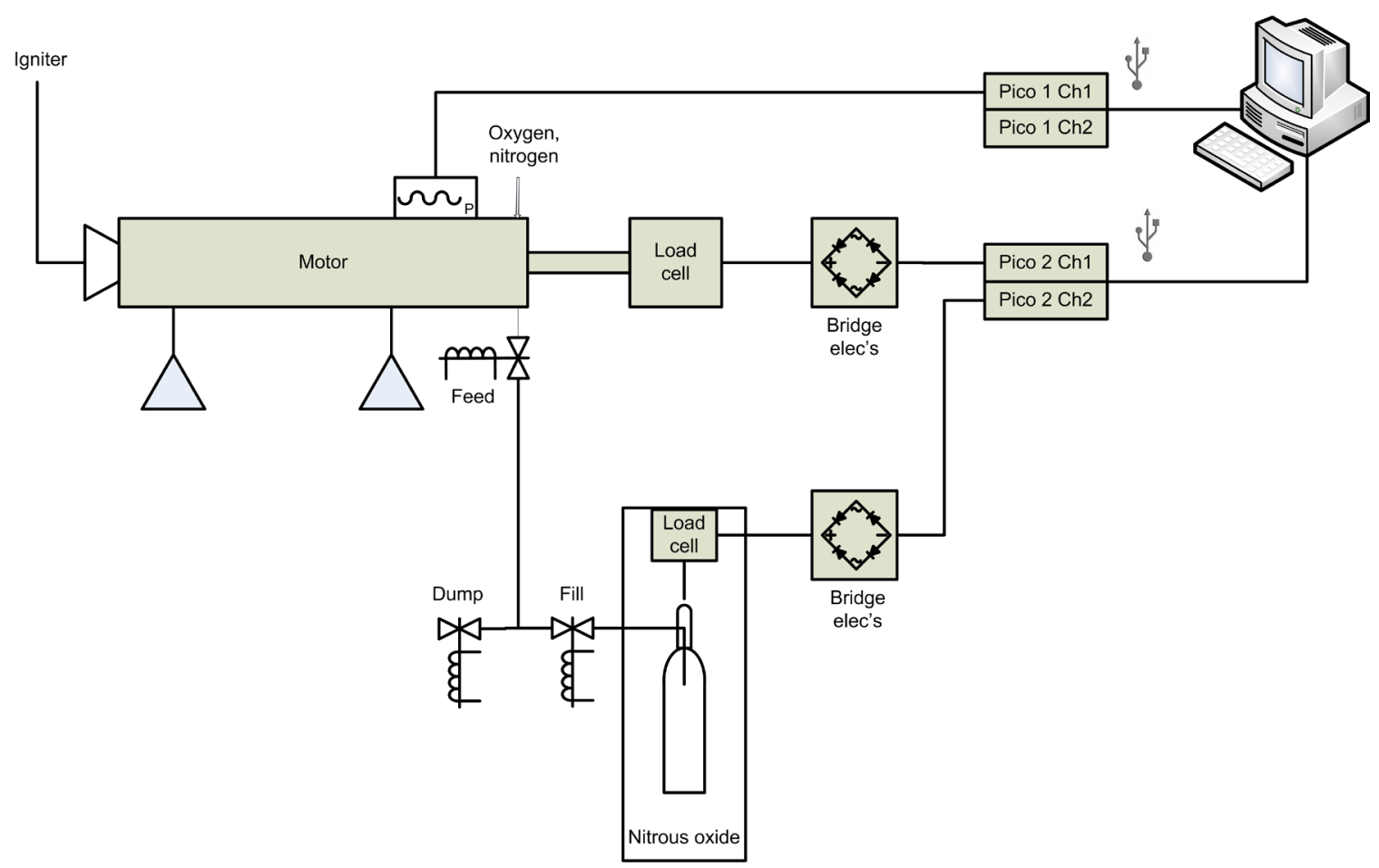

Figure 4. Test schematic 
this load cell was logged at the same time and rate as the thrust load cell, giving a direct correlation between thrust and massflow rate. This simple setup gives a direct reading of mass (weight) flow rate, and does not involve passing liquefied nitrous oxide through orifices which may induce cavitation - nitrous oxide shows strong tendencies to cavitate at laboratory temperature. ${ }^{9}$ In addition, the density of nitrous oxide at ambient temperatures varies considerably, but this does not affect a direct weightmeasuring technique.

The combustion chamber wall is deliberately oversized in section $(10 \mathrm{~mm}$ wall thickness), which allows sufficient depth to provide tapped holes of reasonable length and diameter. For these tests, a pressure sensor was fitted close to the forward end of the combustion chamber (where temperatures are sufficiently low to avoid damage because

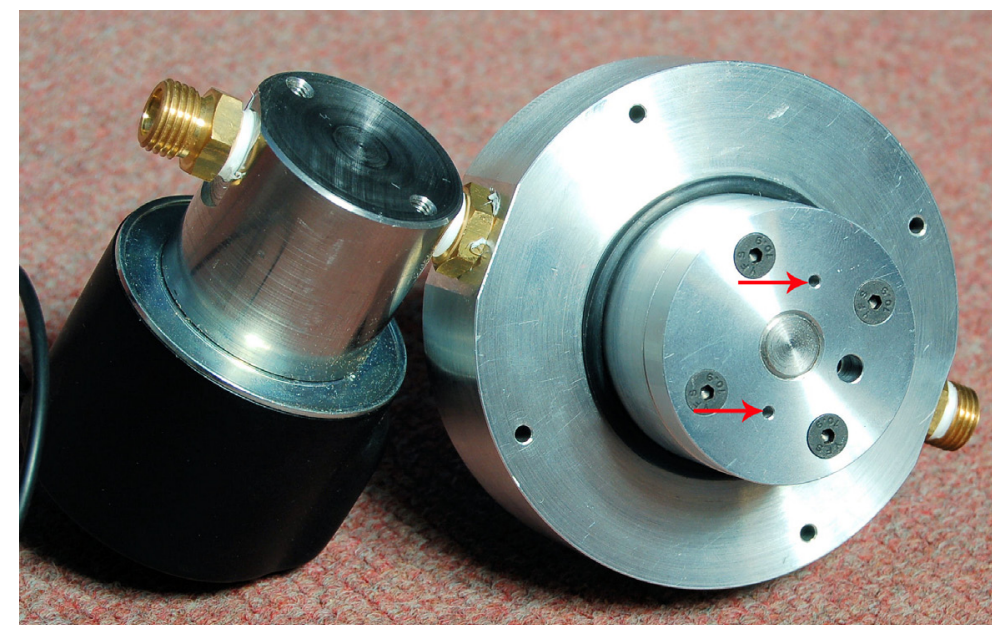

Figure 5. Forward closure with axial injector fitted. Original swirl injector is similar but with injector ports set at $42^{\circ}$ to motor axis. Injector ports (arrowed) are $2 \mathrm{~mm}$ diameter; larger port is for oxygen (ignition) and nitrogen (purge). the nitrous oxide is still vaporising).

All of the instrumentation was connected via a number of BNC terminals on the wall of the test cell to a similar panel close to the cell control panel. A pair of two-channel PicoScope 2203 A-D converters were connected to a PC, and the software supplied with these instruments was used to capture load-cell and pressure-sensor data. This could be viewed graphically, and was also be exported in CSV format to an Excel spreadsheet for further data reduction.

It was anticipated that the burning process would leave clearly visible striations on the grain surface. This would allow the flow angles to be determined easily, aiding a fuller understanding of the processes taking place. This phenomenon has been observed in other test motors, such as the SSTL hybrid. The nitrogen purge capability used was intended to limit any degradation of this detail post-firing, and was successful in this test programme.

\section{Test Procedure}

The motor load cell, flexures and data-capture arrangement were calibrated by hanging weights on a lanyard attached to points adjacent to the load-cell mounting. Weights were progressively added, then progressively removed to check for hysteresis and repeatability.

Each fuel grain was prepared by drilling a $2 \mathrm{~mm}$ diameter hole approximately $10 \mathrm{~mm}$ from the head end for the pressure sensor. Grains were then weighed before assembly into the motor. The motor was assembled with the relevant fuel grain and injector for the configuration to be tested. The test setup was as shown in Figure 4. The PicoLog datacapture software was used to capture samples from all three data channels simultaneously, giving time-synchronised readings. Since relative rather than absolute values for the oxidiser weight were required, an offset was set in the oxidiser weight load cell electronics to remove most of the dead weight and maximise sample resolution. A video camera within the test cell was used to monitor firings via an LCD TV screen, and the video was recorded onto a builtin hard drive for later review.

The motor was ignited by introducing gaseous oxygen then initiating a pyrotechnic igniter ('Davey match'). After 2-3 seconds, the nitrous oxide was introduced at full flow, and the oxygen flow discontinued immediately that full burn was seen. Thrust, chamber pressure and oxidiser cylinder weight were sampled throughout the firing, at a capture rate of 100 samples per second. Each firing was limited to approximately six-seven seconds in duration, to ensure the grain did not completely burn through and risk damage to the motor.

At the end of the burn time, the purge switch was operated, which simultaneously switched off the flow of oxidiser and introduced gaseous nitrogen. This ensured near-instant shutdown, and cooled the motor internals as quickly as possible to preserve any marking of the fuel-grain surface. Purging also prevented burning on using atmospheric oxygen, and also melting of the fuel-grain surface, which would impede accurate physical regression measurements.

After weighing the grain, it was sectioned on a bandsaw, measured, and photographed to record any surface 
markings that indicated gas-flow direction. An example can be seen in Figure 9.

\section{A. Post-Firing Data Capture}

The data are stored by the software in proprietary format, but were exported as CSV files and imported into Excel for analysis. For each firing, four data items per sample were recorded - time in ms, and thrust, pressure and nitrousoxide mass in volts. The relevant section of data, with suitable periods before and after the firing, was identified and pasted into a spreadsheet. Zero values were identified and subtracted from each data point, and the calibration factor for each parameter was then applied. This gave columns for time in milliseconds, thrust in newtons, pressure in bar and nitrous-oxide mass in $\mathrm{kg}$, all referenced to the initial conditions at ignition. These parameters were then displayed on a graph, and the precise firing duration was obtained. Comparing before-and-after masses for the oxidiser, and for the fuel grain, permitted the mass-flow rates and the oxidiser/fuel ratio to be determined.

\section{Problems Encountered}

It was anticipated that at least one shakedown firing would be required, and that some problems would need to be resolved. A number of problems came to light, including poor resolution with instrumentation, in particular the oxidiser mass (and mass-flow rate). Five firings, including some repeated attempts, were carried out before it was decided that good data were being produced. Once solved, the series of firings reported here was begun.

With the axial injector, the motor was found to ignite easily and reliably. However, when the swirling injector was fitted with a straight (non-tapered) fuel grain, the motor immediately extinguished when the nitrous oxide was introduced. Several attempts were made without success. To assist with ignition, two Pyrodex pellets were attached to the electric igniter. The motor fired, but extinguished after about half a second - presumably once the Pyrodex had burned away. It was believed that the liquid nitrous oxide impinging directly onto the fuel-grain surface was cooling the grain and preventing it pyrolysing.

A replacement injector (version 2) was made, with the injector ports much closer to the centre of the injector: $4 \mathrm{~mm}$ apart. Due to the method of mounting the injector to the forward closure, this necessitated adding a $15 \mathrm{~mm}$-high turret to the existing layout (see Fig. 6); this moved the injector face down the fuel-grain port. This turret was slightly smaller in diameter than the port of the straight (non-tapered) grain. This configuration was fired successfully with a straight (nontapered grain. After sectioning the fuel grain, there was deep scoring in the surface where the oxidiser had impinged but no evidence of swirling flow, and it was concluded that the ports were now too close to the centre to impart sufficient tangential velocity. A further two injector ports were drilled, $14 \mathrm{~mm}$ apart. The existing ports were plugged with epoxy resin. When this configuration (version 3) was fired, the thrust was noticeably reduced compared to previous firings, accompanied by a reduction in mass flow. After the firing, a strip examination revealed that the epoxy plugs had been

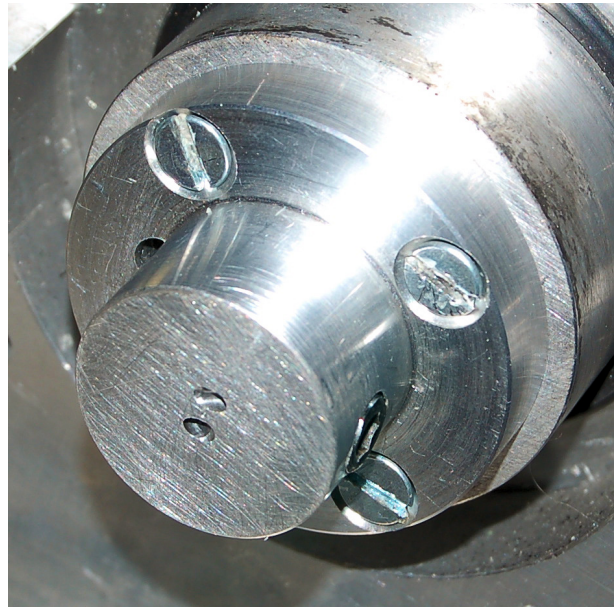

Figure 6. Version 2 of injector. Injector ports are 4 mm apart. Note that a turret has been added to avoid the threaded hole used to attach the injector to the forward closure.

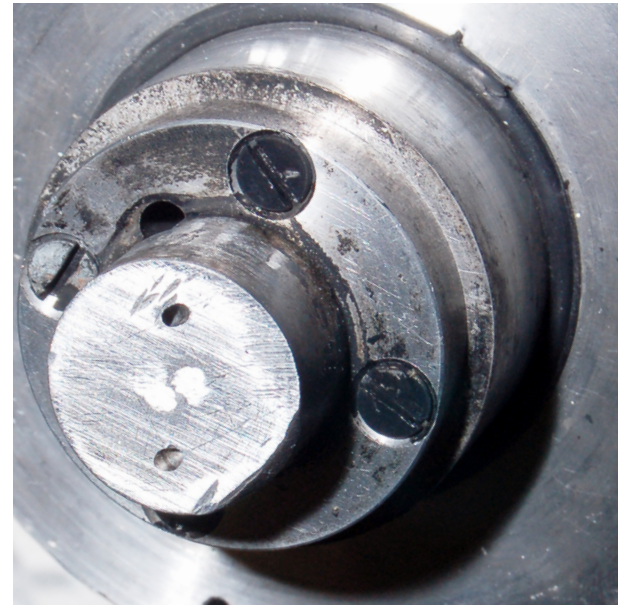

Figure 7. Version 4 of injector. Injector ports are $14 \mathrm{~mm}$ apart. Inner ports have been filled with oversized alloy plugs pressed into place. 
Table 1. Summary of firing data

\begin{tabular}{lllcccccc}
\hline \hline No & Grain & Injector & $\begin{array}{c}\text { Thrust } \\
(\mathrm{N})\end{array}$ & $\begin{array}{c}\text { Burn time } \\
(\mathrm{s})\end{array}$ & O/F ratio & $\begin{array}{c}\text { Total mass-flow } \\
\text { rate }(\mathrm{kg} / \mathrm{s})\end{array}$ & $\begin{array}{c}\text { Regression rate } \\
(\mathrm{mm} / \mathrm{s})\end{array}$ & Isp (s) \\
\hline $3 \mathrm{c}$ & straight & swirl v3* & $103^{\dagger}$ & 6.7 & 5.98 & 0.0637 & 1.66 & 165 \\
8 & taper & swirl v1 & 209 & 8.5 & 9.19 & 0.10772 & 1.76 & 198 \\
9 & straight & axial & 177 & 7.4 & 11.84 & 0.1233 & 1.68 & 148 \\
10 & taper & swirl v4 & 202 & 6.4 & 10.33 & 0.1208 & 1.90 & 170 \\
11 & straight & swirl v4 & 192 & 8.8 & 7.93 & 0.1037 & 1.87 & 188 \\
12 & straight & swirl v4 & 199 & 7.8 & 10.0 & 0.1143 & 1.79 & 178 \\
13 & straight & axial & $170^{\mathbb{1}}$ & 7.2 & 10.8 & 0.1093 & 1.66 & 159 \\
14 & straight & axial & 164 & 6.8 & 12.96 & 0.115 & 1.54 & 146 \\
15 & taper & axial & 154 & 6.6 & 14.6 & 0.117 & 1.43 & 134 \\
16 & taper & axial & 159 & 7.3 & 13.4 & 0.111 & 1.44 & 146 \\
\hline \hline
\end{tabular}

* modified injector but low flow rate observed

${ }^{\dagger}$ thrust fell when oxygen switched off due to low $\mathrm{N}_{2} \mathrm{O}$ flow rate. Lower thrust value used. Plugs ejected from injector during firing so no definite swirl evident.

$\stackrel{*}{*}$ original swirling injector

$\S$ final swirling injector

II significant change in zero thrust reading after firing

ejected. Further examination showed a constriction in the feed hole to the injector ports by a blanking plug.

Aluminium alloy plugs were made and press-fitted to the unused injector ports, the feed port was drilled out from $3 \mathrm{~mm}$ to $4 \mathrm{~mm}$, and the blanking plug was modified. After refitting to the motor, with a straight fuel grain installed, an unsuccessful firing attempt was made, with the flame again being extinguished by the oxidiser. There is a small gap between the injector turret and the grain port, and another attempt was made, this time ensuring that the igniter was pushed into this gap. This meant that an annulus rich in oxygen was present ahead of the nitrous oxide injection. This setup was fired successfully on this and subsequent occasions.

Whilst the modifications to the injector were being made, the motor was successfully fired with the original swirling injector but with a tapered-port fuel grain. However, it proved impossible to repeat this with another tapered grain, so it was deduced that this configuration was extremely marginal. Using the modified injector with a tapered-port fuel grain was found to provide reliable ignition.

\section{Results}

A total of ten successful firings were made, and are shown in Table 1. The firings represent all four combinations of straight and tapered-port fuel grains and axial and swirling injectors, with two or three firings for each combination.

With the exception of firing $3 \mathrm{c}$, the mass-flow rates are reasonably consistent given that the injector configurations are quite different. The nozzle used for the tests, a commercial nozzle made for amateur hybrid rockets, was sized for a higher mass-flow rate than was used for these tests. However, doubling the mass-flow rates to provide optimum nozzle matching would be likely to increase regression rates significantly, reducing the maximum burn times. Since the same nozzle is used for all firings, it is felt that the convenience of using a commercial product outweighs the benefits of accurate matching available from machining a bespoke nozzle for these tests. The nozzle was replaced once during the firing programme, and is in need of replacement again before further firings are carried out, giving a useful nozzle life of 8-10 firings. The erosion takes place not only in the nozzle throat, as would be expected, but also at the nozzle entry, where it is mismatched to the grain port diameter for most of the burn duration due to regression and the flow passes over quite a small radius. This is a particular issue for the axial injector, where two streams of oxidiser mean that the flow is not axi-symmetric, and the erosion tends to degrade the nozzle symmetry. The degradation within a single 
firing, though, is not believed to have a significant effect on the results, and a cautious approach has been taken to nozzle replacement.

The mean regression rates shown in Table 1 were calculated from the fuel mass lost during the firing and the density of the grain material. The density was determined from the dimensions and mass of a bar of stock material. This method is arguably more accurate than direct measurement of the fuel-grain thickness after firing, since it is difficult to account for local variations in thickness, especially for the swirl cases where the grain surface was heavily gouged. It can be seen from the table that regression rate varied from under $1.4 \mathrm{~mm} / \mathrm{s}$ to $1.9 \mathrm{~mm} / \mathrm{s}$. The highest rates are generally associated with swirling injection, although there is a small overlap with the results from the axial-injection firings. The large increases in regression rates found in other studies have not yet been seen in this programme.

A graph of specific impulse against oxidiser/fuel ratio is shown in Fig. 8. It can be seen that the firings with the swirling injection are generally towards the left side of the graph, with a lower O/F ratio, and are thus closer to the optimum for this particular motor.

\section{Discussion}

The small number of firings carried out to date, together with the four configurations, mean that too few data samples exist to provide statistical confidence in any conclusions drawn. However, the results appear to indicate that the use of a swirling injector creates an increase in specific impulse, and that this increase is greater when a tapered (reducing) grain port is used. Further, the use of a tapered port with an axial-flow injector reduces specific impulse, indicating that this improvement is due to the swirling injection rather than the tapering port. This observation gives some confidence that the method investigated shows promise, and that further work is worthwhile. These effects may, of course, apply only to the specific configurations tested.

The parameter used to express performance here is specific impulse. An alternative parameter such as characteristic

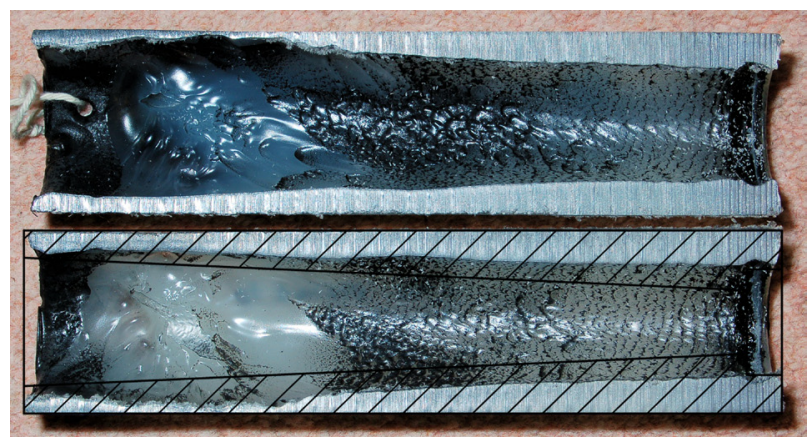

Figure 9. Sectioned fuel grain from firing 10 (swirling injector and tapered fuel grain). Overlaid is the original profile to show regression. The head end is at left. The surface shows evidence of swirling flow along the entire length of the port. Fuel grain is $195 \mathrm{~mm}$ in length.

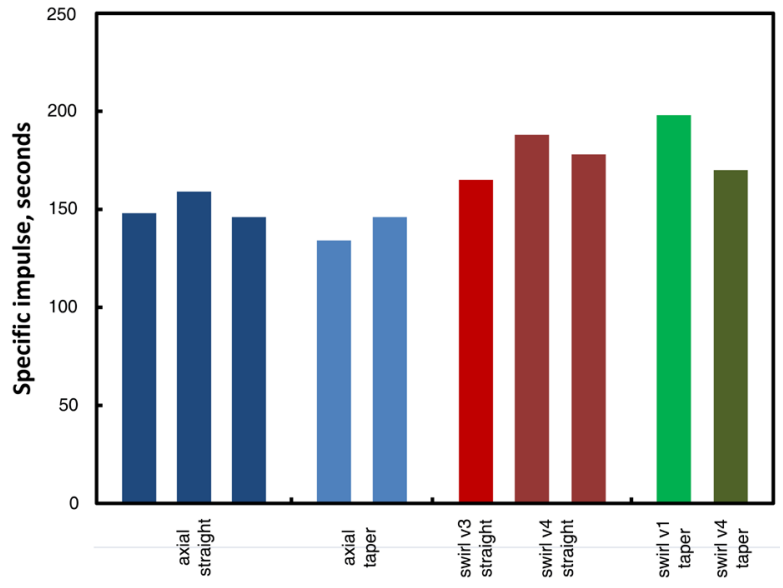

Figure 10. Specific impulse ordered by configuration. 
velocity, $c^{*}$, could have been selected, the benefit being that this parameter is independent of nozzle characteristics, so would take account of any nozzle mismatch to mass-flow rate. Although not reported here, chamber pressure was measured during these firings, and was typically around 6 bar. However, because of the nature of highly swirling flow, the effective nozzle diameter would need to be established for a meaningful value of $c^{*}$ to be calculated. Rotating flow through the nozzle may take a variety of forms with varying density profiles, , so the effective diameter will not be the same as the physical diameter. At this point it is not clear what this will be, and further investigation is required.

Before the firing programme commenced, there was some concern that, whilst the concept of sustained vortex flow may be shown to be effective, the axial regression profile within the grain port may be such as to reduce the taper angle during a firing. This would render the concept ineffective for significant burn durations. The results show this not to be the case, and Fig. 9 shows that the regression is reasonably constant along the length of the port, albeit with local variations due to impingement.

Whilst several papers have been published detailing research using swirling flow based on gaseous oxygen, little published material exists concerning this configuration with liquid oxidisers. The exception is the work carried out by Gibbon and $\mathrm{Haag}^{2}$, but the configuration of the motor was significantly different from the one described here. In this respect, nitrous oxide posed specific difficulties with achieving the correct configuration of injector position relative to the grain surface to avoid flame extinction at start-up. The solution of providing an annular space forward of the injector face in which some pre-combustion with oxygen can occur appears to be a successful one.

\section{Conclusions}

The results obtained to date, although not sufficient to provide statistical confidence, appear to indicate that there is a benefit to be gained from employing swirling flow within a tapered fuel-grain cavity. Further work is required, however, to determine the extent of this benefit and to demonstrate that it is a statistically valid effect. The results indicate that an improvement in specific impulse of the order of $10 \%$ might be expected, together with a small but useful increase in regression rate. The tests have not shown increases in regression rate comparable to the work of others using gaseous oxygen, which in some cases have increased by a factor of five or more.

There is a considerable difference between gaseous oxygen and liquid nitrous oxide, in terms of the complexity when used in a swirling-flow configuration. Whilst accommodating a liquid oxidiser is not a great technical challenge, some limitations in terms of the angle and radial location of the injection ports have been found, and these in turn may restrict the practical configurations that may be achieved.

Further testing is now planned to provide more-detailed characterisation of the behaviour of various fuel-grain geometries with different injection angles and port taper ratios.

\section{References}

${ }^{1}$ Lee, C., Na, Y., and Lee, G., "The Enhancement of Regression Rate of Hybrid Rocket Fuel by Helical Grain Configuration and Swirl Flow," 4lst AIAA/ASME/SAE/ASEE Joint Propulsion Conference and Exhibit, Tucson, USA, 2005.

${ }^{2}$ Gibbon, D. M., and Haag, G.S., "Investigation of an Alternative Geometry Hybrid Rocket for Small Spacecraft Orbit Transfer.” 2001, SSTL. p. 47.

${ }^{3}$ Knuth, W. H., Chiaverini, M. J., Sauer, J. A., Gramer, D. J., "Solid-Fuel Regression Rate Behavior of Vortex Hybrid Rocket Engines," Journal of Propulsion and Power, 2002, 18(3), pp. 600-609.

${ }^{4}$ Knuth, W.H., Chiaverini, M. J., Gramer, D. J., Sauer, J. A., "Experimental Investigation of a Vortex-Driven High-Regression Rate Hybrid Rocket Engine," 34th AIAA/ASME/SAE/ASEE Joint Propulsion Conference and Exhibit, Cleveland, USA, 1998.

${ }^{5}$ Majdalani, J. and Vyas, A. B, "Rotational Axisymmetric Mean Flow for the Vortex Injection Hybrid Rocket Engine." 40th AIAA/ASME/SAE/ASEE Joint Propulsion Conference and Exhibit. Fort Lauderdale, USA, 2004.

${ }^{6}$ Kitoh, O., "Experimental Study of Turbulent Swirling Flow in a Straight Pipe," Journal of Fluid Mechanics, 1991. 225, pp. 445-479.

${ }^{7}$ Steenbergen, W. and J. Voskamp, "The Rate of Decay of Swirl in Turbulent Pipe Flow," Flow Measurement and Instrumentation, 1998, 9(2), pp. 67-78.

${ }^{8}$ Marxman, G.A. and Gilbert, M., "Turbulent Boundary Layer Combustion in the Hybrid Rocket," 9th International Symposium on Combustion, Ithaca, NY, 1962, pp. 371-383.

${ }^{9}$ Thicksten, Z., Macklin, F., and Campbell, J., "Handling Considerations of Nitrous Oxide in Hybrid Rocket Motor Testing," 44th AIAA/ASME/SAE/ASEE Joint Propulsion Conference \& Exhibit, Hartford, USA, 2008. 